\title{
AVERAGE OF COMPLETE JOINT WEIGHT ENUMERATORS AND SELF-DUAL CODES
}

\author{
HIMADRI SHEKHAR CHAKRABORTY* AND TSUYOSHI MIEZAKI
}

\begin{abstract}
In this paper, we give a representation of the average of complete joint weight enumerators of two linear codes of length $n$ over $\mathbb{F}_{q}$ and $\mathbb{Z}_{k}$ in terms of the compositions of $n$ and their distributions in the codes. We also obtain a generalization of the representation for the average of $g$-fold complete joint weight enumerators of codes over $\mathbb{F}_{q}$ and $\mathbb{Z}_{k}$. Finally, the average of intersection numbers of a pair of Type III (resp. Type IV) codes, and its second moment are found.
\end{abstract}

\section{INTRODUCTION}

The notion of the joint weight enumerator of two $\mathbb{F}_{q}$-linear codes was introduced in [6]. Further, the notion of the $g$-fold complete joint weight enumerator of $g$ linear codes over $\mathbb{F}_{q}$ was given in [11]. In recent few years, there has been interest in studying linear codes over the finite rings $\mathbb{Z}_{k}$ of integers modulo $k(k \geq 2)$. The concept of the $g$ fold joint weight enumerator and the $g$-fold multi-weight enumerator of codes over $\mathbb{Z}_{k}$ was investigated in [5]. Furthermore, the average of joint weight enumerators of two binary codes was investigated in [12] using the ordinary weight distributions of the codes, and also their average intersection number was obtained. Inspired by the relation pointed out in [8] between the complete cycle index and the complete weight enumerator, an analogue of the relation between the average complete joint cycle index and the average complete joint weight enumerator of codes was given in [3]. Consecutively, the concept of the average complete joint Jacobi polynomial of codes over $\mathbb{F}_{q}$ and $\mathbb{Z}_{k}$ was defined in [2], and an analogue of the main theorem in [12] was given. In this paper, we define the average complete joint weight enumerator of two linear codes over $\mathbb{F}_{q}$ or $\mathbb{Z}_{k}$, and give a generalization of the main theorem

Date: March 9, 2021.

2010 Mathematics Subject Classification. Primary: 11T71; Secondary: 94B05, $11 \mathrm{~F} 11$.

Key words and phrases. Codes, weight enumerators, equivalence.

*Corresponding author. 
in [12 for it. Moreover, we extend the idea of the average complete joint weight enumerator to the average of $g$-fold complete joint weight enumerators of linear codes over $\mathbb{F}_{q}$ or $\mathbb{Z}_{k}$. We take the average on all permutationally (not monomially) equivalent linear codes over $\mathbb{F}_{q}$ or $\mathbb{Z}_{k}$. In [13], the average of intersection numbers of a pair of Type I or Type II codes over $\mathbb{F}_{2}$ and their second moments were given. As a part of our study, we give the average of intersection numbers and its second moment of a pair of Type III codes over $\mathbb{F}_{3}$ as well as Type IV codes over $\mathbb{F}_{4}$.

Throughout this paper, we assume that $R$ denotes either the finite field $\mathbb{F}_{q}$ of order $q$, where $q$ is a prime power or the ring $\mathbb{Z}_{k}$ of integers modulo $k$ for some integer $k \geq 2$. Let $u=\left(u_{1}, u_{2}, \ldots, u_{n}\right)$ and $v=$ $\left(v_{1}, v_{2}, \ldots, v_{n}\right)$ be the elements of $R^{n}$. Then the inner product of $u, v \in$ $R^{n}$ is given by

$$
u \cdot v:=u_{1} v_{1}+u_{2} v_{2}+\cdots+u_{n} v_{n}
$$

If $u \cdot v=0$, we call $u$ and $v$ orthogonal. An element $u \in R^{n}$ is called self-orthogonal if $u \cdot u=0$. When we consider a vector space over $\mathbb{F}_{4}$, it is convenient to consider another inner product given by $u \cdot v:=$ $u_{1} \bar{v}_{1}+u_{2} \bar{v}_{2}+\cdots+u_{n} \bar{v}_{n}$, where $\bar{a}=a^{2}$ for $a \in \mathbb{F}_{4}$.

An $\mathbb{F}_{q^{-}}$-linear code of length $n$ is a vector subspace of $\mathbb{F}_{q}^{n}$, and a $\mathbb{Z}_{k^{-}}$ linear code of length $n$ is an additive group of $\mathbb{Z}_{k}^{n}$. Let $C$ be an $R$-linear code of length $n$. The elements of $C$ are called codewords. The dual code of $C$ is defined as

$$
C^{\perp}:=\left\{v \in R^{n} \mid u \cdot v=0 \text { for all } u \in C\right\}
$$

If $C \subseteq C^{\perp}$, then $C$ is called self-orthogonal, and if $C=C^{\perp}$, then $C$ is called self-dual. Clearly, if $C$ is self-dual, every codeword $u \in C$ is self-orthogonal.

It is well known that the length $n$ of a self-dual code over $\mathbb{F}_{q}$ is even and the dimension is $n / 2$. A self-dual code $C$ over $\mathbb{F}_{2}$ or $\mathbb{F}_{4}$ of length $n \equiv 0(\bmod 2)$ having even weight is called Type I or Type IV, respectively. A self-dual code $C$ over $\mathbb{F}_{2}$ of length $n \equiv 0(\bmod 8)$ is called Type II if the weight of each codeword of $C$ is a multiple of 4 . Finally, a self-dual code $C$ over $\mathbb{F}_{3}$ of length $n \equiv 0(\bmod 4)$ is called Type III if the weight of each codeword of $C$ is a multiple of 3 .

Let the elements of $R$ be $0=\omega_{0}, \omega_{1}, \ldots, \omega_{|R|-1}$ in some fixed order. Then the composition of an element $u \in R^{n}$ is defined as

$$
\operatorname{comp}(u):=s(u):=\left(s_{0}(u), s_{1}(u), \ldots, s_{|R|-1}(u)\right)
$$


where $s_{i}(u)$ denotes the number of coordinates of $u$ that are equal to $\omega_{i}$. Obviously

$$
\sum_{i=0}^{|R|-1} s_{i}(u)=n .
$$

In general, a composition $s$ of $n$ is a vector $s=\left(s_{0}, s_{1}, \ldots, s_{|R|-1}\right)$ with non-negative integer components such that

$$
\sum_{i=0}^{|R|-1} s_{i}=n
$$

Let $C$ be an $R$-linear code of length $n$. We denote by $T_{s}^{C}$ the set of codewords of $C$ with composition $s$ and by $A_{s}^{C}:=\left|T_{s}^{C}\right|$, that is, the number of codewords $u \in C$ such that $\operatorname{comp}(u)=s$. Then the complete weight enumerator of $C$ is defined as:

$$
\begin{aligned}
\mathcal{C}_{C}\left(x_{0}, \ldots, x_{|R|-1}\right) & :=\sum_{u \in C} x_{0}^{s_{0}(u)} \ldots x_{|R|-1}^{s_{|R|-1}(u)} \\
& =\sum_{s} A_{s}^{C} x_{0}^{s_{0}} \ldots x_{|R|-1}^{s_{|R|-1}}
\end{aligned}
$$

where $x_{0}, \ldots, x_{|R|-1}$ are indeterminates and the sum extends over all compositions $s$ of $n$. Let $K:=\{0,1, \ldots,|R|-1\}$. Then the complete weight enumerator of $C$ can be written as:

$$
\mathcal{C}_{C}\left(x_{i} \text { with } i \in K\right)=\sum_{s} A_{s}^{C} \prod_{i=0}^{|R|-1} x_{i}^{s_{i}} .
$$

Now let $C$ and $D$ be two $R$-linear codes of length $n$. We denote by $\eta(u, v)$ the bi-composition of the pair $(u, v)$ for $u, v \in R^{n}$ which is a vector with non-negative integer components $\eta_{\alpha \beta}(u, v)$ defined as

$$
\eta_{\alpha \beta}(u, v):=\#\left\{i \mid\left(u_{i}, v_{i}\right)=(\alpha, \beta)\right\}
$$

where $(\alpha, \beta) \in R^{2}$. Clearly

$$
\sum_{\alpha, \beta \in R} \eta_{\alpha \beta}(u, v)=n
$$

In general, a bi-composition $\eta$ of $n$ is a vector with non-negative integer components $\eta_{\alpha \beta}$ such that

$$
\sum_{\alpha, \beta \in R} \eta_{\alpha \beta}=n
$$


The complete joint weight enumerator of $C$ and $D$ is defined as

$$
\begin{aligned}
\mathcal{C J}_{C, D}\left(x_{i j} \text { with } i, j \in K\right) & :=\sum_{u \in C, v \in D} \prod_{i, j=0}^{|R|-1} x_{i j}^{\eta_{\omega_{i} \omega_{j}}(u, v)} \\
& =\sum_{\eta} A_{\eta}^{C, D} \prod_{i, j=0}^{|R|-1} x_{i j}^{\eta_{\omega_{i} \omega_{j}}}
\end{aligned}
$$

where $x_{i j}$ for $i, j \in K$ are the indeterminates and $A_{\eta}^{C, D}$ is the number of pair $(u, v) \in C \times D$ such that $\eta(u, v)=\eta$.

We write $\mathcal{S}_{n}$ for the symmetric group acting on the set $\{1,2, \ldots, n\}$, equipped with the composition of permutations. For any $R$-linear code $C$, the code $C^{\sigma}:=\left\{u^{\sigma} \mid u \in C\right\}$ for some permutation $\sigma \in \mathcal{S}_{n}$ is called permutationally equivalent to $C$, where $u^{\sigma}:=\left(u_{\sigma(1)}, \ldots, u_{\sigma(n)}\right)$. Then the average complete joint weight enumerator of $R$-linear codes $C$ and $D$ is defined as

$$
\mathcal{C} \mathcal{J}_{C, D}^{a v}\left(x_{i j} \text { with } i, j \in K\right):=\frac{1}{n !} \sum_{\sigma \in \mathcal{S}_{n}} \mathcal{C} \mathcal{J}_{C^{\sigma}, D}\left(x_{i j}\right) .
$$

This paper is organized as follows. In Section 2, we give the average version of the MacWilliams identity for the complete joint weight enumerators. In Sections 3, we give the main result (Theorem 3.1) of this paper, and in Section 4, we obtain a generalization of Theorem 3.1 for the average of $g$-fold complete joint weight enumerators of codes over $\mathbb{F}_{q}$ and $\mathbb{Z}_{k}$ (Theorem 4.1). In Section 5, the average of intersection numbers of a pair of Type III (resp. Type IV) codes, and its second moment are given (Theorem 5.4 and Theorem 5.5).

\section{MacWilliams Identity}

The MacWilliams identity for $g$-fold complete joint weight enumerators of codes over $\mathbb{F}_{q}$ was established in [11]. Further, in [5], the MacWilliams identity for $g$-fold joint weight enumerators of codes over $\mathbb{Z}_{k}$ was given. In this section, we study the MacWilliams type identity for the average complete joint enumerators over $R$. At the beginning of this section we recall [5, 6] to take some fixed character over $R$.

A character $\chi$ of $R$ is a homomorphism from the additive group $R$ to the multiplicative group of non-zero complex numbers.

Let $R=\mathbb{F}_{q}$, where $q=p^{f}$ for some prime number $p$. Again let $F(x)$ be a primitive irreducible polynomial of degree $f$ over $\mathbb{F}_{p}$ and let $\lambda$ be a root of $F(x)$. Then any element $\alpha \in \mathbb{F}_{q}$ has a unique representation 
as:

$$
\alpha=\alpha_{0}+\alpha_{1} \lambda+\alpha_{2} \lambda^{2}+\cdots+\alpha_{f-1} \lambda^{f-1}
$$

where $\alpha_{i} \in \mathbb{F}_{p}$, and $\chi(\alpha):=\zeta_{p}^{\alpha_{0}}$, where $\zeta_{p}$ is the primitive $p$-th root $e^{2 \pi i / p}$ of unity, and $\alpha_{0}$ is given by (11).

Again if $R=\mathbb{Z}_{k}$, then for $\alpha \in \mathbb{Z}_{k}$ we defined $\chi$ as $\chi(\alpha):=\zeta_{k}^{\alpha}$, where $\zeta_{k}$ is the primitive $k$-th root $e^{2 \pi i / k}$ of unity.

We have the MacWilliams identity for the complete weight enumerator of a code $C$ over $R$ as follows.

Theorem $2.1([5,6])$. For a code $C$ over $R$ we have

$$
\mathcal{C}_{C^{\perp}}\left(x_{i} \text { with } i \in K\right)=\frac{1}{|C|} T_{R} \cdot \mathcal{C}_{C}\left(x_{i}\right)
$$

where $T_{R}=(\chi(\alpha \beta))_{\alpha, \beta \in R}$.

For a code $C$ over $R$ let $\tilde{C}$ be either $C$ or $C^{\perp}$. Then we define

$$
\delta(C, \tilde{C}):=\left\{\begin{array}{lll}
0 & \text { if } \quad \tilde{C}=C \\
1 & \text { if } \quad \tilde{C}=C^{\perp}
\end{array}\right.
$$

For $\alpha \in R$, we define $\gamma(\alpha):=j$ if $\alpha=\omega_{j}$. Though it is obvious for the experts, we give a proof of the following Theorem for general readers.

Theorem 2.2 (MacWilliams Identity). Let $C$ and $D$ be two R-linear codes of length $n$. Then we have

$\mathcal{C} \mathcal{J}_{\tilde{C}, \tilde{D}}\left(x_{i j}\right.$ with $\left.i, j \in K\right)=\frac{1}{|C|^{\delta(C, \tilde{C})}|D|^{\delta(D, \tilde{D})}} T_{R}^{\delta(C, \tilde{C})} \otimes T_{R}^{\delta(D, \tilde{D})} \mathcal{C} \mathcal{J}_{C, D}\left(x_{i j}\right)$.

Proof. It is sufficient to show

$$
|D| \mathcal{C} \mathcal{J}_{C, D^{\perp}}\left(x_{i j}\right)=\left(I \otimes T_{R}\right) \mathcal{C} \mathcal{J}_{C, D}\left(x_{i j}\right)
$$

where, $\tilde{C}=C, \tilde{D}=D^{\perp}$, and $I$ is the identity matrix. Let

$$
\delta_{D^{\perp}}(v):= \begin{cases}1 & \text { if } \quad v \in D^{\perp} \\ 0 & \text { otherwise }\end{cases}
$$

Then we have the following identity

$$
\delta_{D^{\perp}}(v)=\frac{1}{|D|} \sum_{d \in D} \chi(d \cdot v)
$$


Now

$$
\begin{aligned}
& |D| \mathcal{C} \mathcal{J}_{C, D^{\perp}}\left(x_{i j}\right)=|D| \sum_{c \in C} \sum_{d^{\prime} \in D^{\perp}} \prod_{i, j=0}^{|R|-1} x_{i j}^{\eta_{\omega_{i} \omega_{j}}\left(c, d^{\prime}\right)} \\
& =|D| \sum_{c \in C} \sum_{v \in R^{n}} \delta_{D^{\perp}}(v) \prod_{i, j=0}^{|R|-1} x_{i j}^{\eta_{\omega_{i} \omega_{j}}(c, v)} \\
& =\sum_{c \in C} \sum_{v \in R^{n}} \sum_{d \in D} \chi(d \cdot v) \prod_{i, j=0}^{|R|-1} x_{i j}^{\eta_{\omega_{i} \omega_{j}}(c, v)} \\
& =\sum_{c \in C, d \in D} \sum_{v \in R^{n}} \chi(d \cdot v) \prod_{i, j=0}^{|R|-1} x_{i j}^{\eta_{\omega_{i} \omega_{j}}(c, v)} \\
& =\sum_{c \in C, d \in D} \sum_{\left(v_{1}, \ldots, v_{n}\right) \in R^{n}} \chi\left(d_{1} v_{1}+\cdots+d_{n} v_{n}\right) \prod_{1 \leq i \leq n} x_{\gamma\left(c_{i}\right) \gamma\left(v_{i}\right)} \\
& =\sum_{c \in C, d \in D} \prod_{1 \leq i \leq n} \sum_{v_{i} \in R} \chi\left(d_{i} v_{i}\right) x_{\gamma\left(c_{i}\right) \gamma\left(v_{i}\right)} \\
& =\sum_{c \in C, d \in D} \prod_{(\alpha, \beta) \in R^{2}}\left(\sum_{v \in R} \chi(\beta v) x_{\gamma(\alpha) \gamma(v)}\right)^{\eta_{\alpha \beta}(c, d)} \\
& =\mathcal{C} \mathcal{J}_{C, D}\left(\sum_{v \in R} \chi(\beta v) x_{\gamma(\alpha) \gamma(v)} \text { with }(\alpha, \beta) \in R^{2}\right) \\
& =\left(I \otimes T_{R}\right) \mathcal{C} \mathcal{J}_{C, D}\left(x_{i j}\right) \text {. }
\end{aligned}
$$

Hence, the proof is completed.

Now from the above Theorem 2.2, we have the generalized MacWilliams identity for the average complete joint weight enumerator of codes $C$ and $D$ as follows:

$\mathcal{C} \mathcal{J}_{\tilde{C}, \tilde{D}}^{a v}\left(x_{i j}\right.$ with $\left.i, j \in K\right)=\frac{1}{|C|^{\delta(C, \tilde{C})}|D|^{\delta(D, \tilde{D})}} T_{R}^{\delta(C, \tilde{C})} \otimes T_{R}^{\delta(D, \tilde{D})} \mathcal{C} \mathcal{J}_{C, D}^{a v}\left(x_{i j}\right)$.

\section{Main Result}

In this section, we give the main result of this paper which is presented in the following theorem.

Theorem 3.1 (Main Theorem). Let $C$ and $D$ be two $R$-linear codes of length $n$, and $r$ and $s$ be the compositions of $n$. Again let $\eta$ be the 
bi-composition of $n$ such that

$$
\begin{aligned}
r & =\left(\sum_{\beta \in R} \eta_{\omega_{0} \beta}, \ldots, \sum_{\beta \in R} \eta_{\omega_{|R|-1} \beta}\right), \\
s & =\left(\sum_{\alpha \in R} \eta_{\alpha \omega_{0}}, \ldots, \sum_{\alpha \in R} \eta_{\alpha \omega_{|R|-1}}\right) .
\end{aligned}
$$

Then we have

$$
\begin{aligned}
& \mathcal{C J}_{C, D}^{a v}\left(x_{i j} \text { with } i, j \in K\right) \\
&=\sum_{r, s, \eta} A_{r}^{C} A_{s}^{D} \frac{\prod_{i=0}^{|R|-1}\left(\begin{array}{c}
s_{i} \\
\eta_{\omega_{0} \omega_{i}}, \ldots, \eta_{\omega_{|R|-1} \omega_{i}}
\end{array}\right)}{\left(\begin{array}{c}
n \\
r_{0}, \ldots, r_{|R|-1}
\end{array}\right)} \prod_{i, j=0}^{|R|-1} x_{i j}^{\eta_{\omega_{i} \omega_{j}}},
\end{aligned}
$$

where

$$
\left(\begin{array}{c}
a \\
b_{0}, b_{1}, \ldots, b_{m}
\end{array}\right):=\frac{a !}{b_{0} ! b_{1} ! \ldots b_{m} !} .
$$

Proof. Let $C$ and $D$ be two $R$-linear codes of length $n$. Then the complete joint weight enumerator of $C$ and $D$ is

$$
\mathcal{C} \mathcal{J}_{C, D}\left(x_{i j} \text { with } i, j \in K\right):=\sum_{\eta} A_{\eta}^{C, D} \prod_{i, j=0}^{|R|-1} x_{i j}^{\eta_{\omega_{i} \omega_{j}}}
$$

where $\sum_{\alpha, \beta \in R} \eta_{\alpha \beta}=n$. Now let us define

$$
B_{r, s, \eta}^{C, D}:=\#\{(u, v) \in C \times D \mid \operatorname{comp}(u)=r, \operatorname{comp}(v)=s, \eta(u, v)=\eta\} .
$$

Therefore, $A_{\eta}^{C, D}=B_{r, s, \eta}^{C, D}$, where

$$
\begin{aligned}
r & =\left(\sum_{\beta \in R} \eta_{\omega_{0} \beta}, \ldots, \sum_{\beta \in R} \eta_{\omega_{|R|-1} \beta}\right), \\
s & =\left(\sum_{\alpha \in R} \eta_{\alpha \omega_{0}}, \ldots, \sum_{\alpha \in R} \eta_{\alpha \omega_{|R|-1}}\right) .
\end{aligned}
$$

Hence, we can write from (2)

$$
\mathcal{C} \mathcal{J}_{C, D}\left(x_{i j} \text { with } i, j \in K\right):=\sum_{r, s, \eta} B_{r, s, \eta}^{C, D} \prod_{i, j=0}^{|R|-1} x_{i j}^{\eta_{\omega_{i} \omega_{j}}}
$$


Now

$$
\begin{aligned}
\sum_{\sigma \in \mathcal{S}_{n}} B_{r, s, \eta}^{C^{\sigma}, D} & =\#\left\{(u, v, \sigma) \in T_{r}^{C} \times T_{s}^{D} \times \mathcal{S}_{n} \mid \eta\left(u^{\sigma}, v\right)=\eta\right\} \\
& =\sum_{u \in T_{r}^{C}} \sum_{v \in T_{s}^{D}} \#\left\{\sigma \in \mathcal{S}_{n} \mid \eta\left(u^{\sigma}, v\right)=\eta\right\}
\end{aligned}
$$

It is well known that the order of a subgroup of $\mathcal{S}_{n}$ which stabilizes $u \in T_{r}^{C}$ is $\prod_{i=0}^{|R|-1} r_{i}$ !. Therefore,

$$
\begin{aligned}
\sum_{\sigma \in \mathcal{S}_{n}} B_{r, s, \eta}^{C^{\sigma}, D} & =\sum_{u \in T_{r}^{C}} \sum_{v \in T_{s}^{D}} \prod_{i=0}^{|R|-1} r_{i} ! \#\left\{u^{\prime} \in R^{n} \mid \operatorname{comp}\left(u^{\prime}\right)=r, \eta\left(u^{\prime}, v\right)=\eta\right\} \\
& =\sum_{u \in T_{r}^{C}} \sum_{v \in T_{s}^{D}} \prod_{i=0}^{|R|-1} r_{i} ! \prod_{i=0}^{|R|-1} \frac{s_{i} !}{\prod_{j=0}^{|R|-1} \eta_{\omega_{j} \omega_{i}} !} \\
& =A_{r}^{C} A_{s}^{D} \prod_{i=0}^{|R|-1} r_{i} ! \prod_{i=0}^{|R|-1} \frac{s_{i} !}{\prod_{j=0}^{|R|-1} \eta_{\omega_{j} \omega_{i}} !} \\
& =A_{r}^{C} A_{s}^{D} n ! \frac{\prod_{i=0}^{|R|-1} \frac{s_{i} !}{\prod_{j=0}^{|R|-1} \eta_{\omega_{j} \omega_{i}} !}}{n !} \\
& =A_{r}^{C} A_{s}^{D} n ! \frac{\prod_{i=0}^{|R|-1} r_{i} !}{\left(\begin{array}{c}
|R|-1 \\
\eta_{\omega_{0} \omega_{i}}, \ldots, \eta_{\omega_{|R|-1} \omega_{i}}
\end{array}\right)}
\end{aligned}
$$

Now we have

$$
\begin{aligned}
\mathcal{C J}_{C, D}^{a v}( & \left.x_{i j} \text { with } i, j \in K\right) \\
= & \frac{1}{n !} \sum_{\sigma \in \mathcal{S}_{n}} \mathcal{C J}_{C^{\sigma}, D}\left(x_{i j}\right) \\
= & \frac{1}{n !} \sum_{r, s, \eta} \sum_{\sigma \in \mathcal{S}_{n}} B_{r, s, \eta}^{C^{\sigma}, D} \prod_{i, j=0}^{|R|-1} x_{i j}^{\eta_{\omega_{i} \omega_{j}}}
\end{aligned}
$$




$$
=\sum_{r, s, \eta} A_{r}^{C} A_{s}^{D} \frac{\prod_{i=0}^{|R|-1}\left(\begin{array}{c}
s_{i} \\
\eta_{\omega_{0} \omega_{i}}, \ldots, \eta_{\omega_{|R|-1} \omega_{i}}
\end{array}\right)}{\left(\begin{array}{c}
n \\
r_{0}, r_{1}, \ldots, r_{|R|-1}
\end{array}\right)} \prod_{i, j=0}^{|R|-1} x_{i j}^{\eta_{\omega_{i} \omega_{j}}} .
$$

This completes the proof.

\section{Average of $g$-Fold Complete Joint Weight ENUMERATORS}

In this section, we give a generalization of the Main Theorem for the average $g$-fold complete joint weight enumerators of codes over $R$.

Let $C_{1}, C_{2}, \ldots, C_{g}$ be $R$-linear codes of length $n$. We denote by $\eta^{g}\left(c_{1}, \ldots, c_{g}\right)$ the $g$-fold composition of $g$-tuple

$$
\left(c_{1}, \ldots, c_{g}\right) \in C_{1} \times \cdots \times C_{g}
$$

which is a vector with non-negative integer components $\eta_{a}^{g}\left(c_{1}, \ldots, c_{g}\right)$ for $a \in R^{g}$ and defined as:

$$
\eta_{a}^{g}\left(c_{1}, \ldots, c_{g}\right):=\#\left\{i \mid\left(c_{1 i}, \ldots, c_{g i}\right)=a\right\} .
$$

We denote by a $g$-fold composition $\eta^{g}$ of $n$ a vector with non-negative integer components $\eta_{a}^{g}$ for $a \in R^{g}$ such that

$$
\sum_{a \in R^{g}} \eta_{a}^{g}=n
$$

We also denote by $T_{\eta^{g}}^{C_{1}, \ldots, C_{g}}$ the set of codewords of $C_{1} \times \cdots \times C_{g}$ with $g$-fold composition $\eta^{g}$. The $g$-fold complete joint weight enumerator is defined as follows:

$$
\begin{aligned}
\mathcal{C} \mathcal{J}_{C_{1}, \ldots, C_{g}}\left(x_{\gamma(a)} \text { with } a \in R^{g}\right) & :=\sum_{c_{1} \in C_{1}, \ldots, c_{g} \in C_{g}} \prod_{a \in R^{g}} x_{\gamma(a)}^{\eta_{a}^{g}\left(c_{1}, \ldots, c_{g}\right)} \\
& =\sum_{\eta^{g}} A_{\eta^{g}}^{C_{1}, \ldots, C_{g}} \prod_{a \in R^{g}} x_{\gamma(a)}^{\eta_{a}^{g}},
\end{aligned}
$$

where $x_{\gamma(a)}$ for $a \in R^{g}$ with $\gamma(a):=\left(\gamma\left(a_{1}\right), \ldots, \gamma\left(a_{g}\right)\right)$ are the indeterminates and $A_{\eta^{g}}^{C_{1}, \ldots, C_{g}}$ is the number of $g$-tuples $\left(c_{1}, \ldots, c_{g}\right) \in$ $C_{1} \times \cdots \times C_{g}$ such that $\eta^{g}\left(c_{1}, \ldots, c_{g}\right)=\eta^{g}$. The average $g$-fold complete joint weight enumerators are defined as:

$$
\mathcal{C} \mathcal{J}_{C_{1}, C_{2}, \ldots, C_{g}}^{a v}\left(x_{\gamma(a)} \text { with } a \in R^{g}\right):=\frac{1}{n !} \sum_{\sigma \in \mathcal{S}_{n}} \mathcal{C} \mathcal{J}_{C_{1}^{\sigma}, C_{2}, \ldots, C_{g}}\left(x_{\gamma(a)}\right) .
$$


Let $a=\left(a_{1}, \ldots, a_{g}\right) \in R^{g}$ and $b=\left(b_{1}, \ldots, b_{g-1}\right) \in R^{g-1}$. Then we denote

$$
\begin{aligned}
& {[a ; j]:=\left(a_{1}, \ldots, a_{j-1}, a_{j+1}, \ldots, a_{g}\right) \in R^{g-1}} \\
& (z ; b):=\left(z, b_{1}, \ldots, b_{g-1}\right) \in R^{g} \text { for } z \in R .
\end{aligned}
$$

Now we have the following generalization of Theorem 3.1 .

Theorem 4.1. Let $C_{1}, C_{2}, \ldots, C_{g}$ be the $R$-linear codes of length $n$ and $s_{1}, s_{2}, \ldots, s_{g}$ be the composition of $n$. Again let $\eta^{g}$ be the $g$-fold composition of $n$ such that

$$
s_{j}=\left(\sum_{a \in R^{g}} \eta_{a}^{g} \text { with } a_{j}=\omega_{i} \text { for } i \in K\right) \quad \text { where } j=1,2, \ldots, g,
$$

and $\eta^{g-1}$ be the $(g-1)$-fold composition of $n$ such that the non-negative integer components $\eta_{b}^{g-1}$ for $b \in R^{g-1}$ is equal to the sum of $\eta_{a}^{g}$ over all $a \in R^{g}$ with $[a ; 1]=b$, that is,

$$
\eta_{b}^{g-1}=\sum_{a \in R^{g}} \eta_{\left.a\right|_{[a ; 1]=b}}^{g} .
$$

Then we have

$$
\begin{aligned}
& \mathcal{C} \mathcal{J}_{C_{1}, \ldots, C_{g}}^{a v}\left(x_{\gamma(a)} \text { with } a \in R^{g}\right) \\
& =\sum_{s_{1}, \eta^{g-1}, \eta^{g}} A_{s_{1}}^{C_{1}} A_{\eta^{g-1}}^{C_{2}, \ldots, C_{g}} \frac{\prod_{b \in R^{g-1}}\left(\begin{array}{c}
\eta_{b}^{g-1} \\
\eta_{\left(\omega_{0} ; b\right)}^{g}, \ldots, \eta_{\left(\omega_{|R|-1}, b\right)}^{g}
\end{array}\right)}{\left(\begin{array}{c}
n \\
s_{10}, \ldots, s_{1|R|-1}
\end{array}\right)} \prod_{a \in R^{g}} x_{\gamma(a)}^{\eta_{a}^{g}},
\end{aligned}
$$

where

$$
\left(\begin{array}{c}
r \\
r_{0}, r_{1}, \ldots, r_{m}
\end{array}\right):=\frac{r !}{r_{0} ! r_{1} ! \ldots r_{m} !}
$$

Proof. Let $C_{1}, \ldots, C_{g}$ be $R$-linear codes of length $n$. Then by the definition of $g$-fold complete joint weight enumerator of the codes $C_{1}, \ldots, C_{g}$ we have,

$$
\mathcal{C} \mathcal{J}_{C_{1}, \ldots, C_{g}}\left(x_{\gamma(a)} \text { with } a \in R^{g}\right):=\sum_{\eta^{g}} A_{\eta^{g}}^{C_{1}, \ldots, C_{g}} \prod_{a \in R^{g}} x_{\gamma(a)}^{\eta_{a}^{g}},
$$

where

$$
\sum_{a \in R^{g}} \eta_{a}^{g}=n
$$

Now let us define

$$
\begin{aligned}
B_{s_{1}, \eta^{g-1}, \eta^{g}}^{C_{1}, \ldots, C_{g}}:=\#\left\{\left(c_{1}, \ldots, c_{g}\right) \in C_{1} \times \cdots \times C_{g} \mid \operatorname{comp}\left(c_{1}\right)=s_{1},\right. \\
\\
\left.\eta^{g-1}\left(c_{2}, \ldots, c_{g}\right)=\eta^{g-1}, \eta^{g}\left(c_{1}, \ldots, c_{g}\right)=\eta^{g}\right\} .
\end{aligned}
$$


Therefore,

$$
A_{\eta^{g}}^{C_{1}, \ldots, C_{g}}=B_{s_{1}, \eta^{g-1}, \eta^{g}}^{C_{1}, \ldots, C_{g}} .
$$

Hence, we can write from (44)

$$
\mathcal{C} \mathcal{J}_{C_{1}, \ldots, C_{g}}\left(x_{\gamma(a)} \text { with } a \in R^{g}\right):=\sum_{s_{1}, \eta^{g-1}, \eta^{g}} B_{s_{1}, \eta^{g-1}, \eta^{g}}^{C_{1}, \ldots, C_{g}} \prod_{a \in R^{g}} x_{\gamma(a)}^{\eta_{a}^{g}} .
$$

Now

$$
\begin{aligned}
& \sum_{\sigma \in \mathcal{S}_{n}} B_{s_{1}, \eta^{g-1}, \eta^{g}}^{C_{1}^{\sigma}, C_{2}, \ldots, C_{g}} \\
= & \#\left\{\left(c_{1}, \ldots, c_{g}, \sigma\right) \in T_{s_{1}}^{C_{1}} \times \cdots \times T_{s_{g}}^{C_{g}} \times \mathcal{S}_{n} \mid \eta^{g}\left(c_{1}^{\sigma}, c_{2}, \ldots, c_{g}\right)=\eta^{g}\right\} \\
= & \sum_{c_{1} \in T_{s_{1}}^{C_{1}}} \sum_{\left(c_{2}, \ldots, c_{g}\right) \in T_{\eta^{g-1}}^{C_{2}, \ldots, C_{g}}} \#\left\{\sigma \in \mathcal{S}_{n} \mid \eta^{g}\left(c_{1}^{\sigma}, c_{2}, \ldots, c_{g}\right)=\eta^{g}\right\} .
\end{aligned}
$$

It is well known that the order of a subgroup of $\mathcal{S}_{n}$ which stabilizes $c_{1} \in T_{s_{1}}^{C_{1}}$ is $\prod_{i=0}^{|R|-1} s_{1 i} !$. Therefore,

$$
\begin{aligned}
\sum_{\sigma \in \mathcal{S}_{n}} B_{s_{1}, \eta^{g-1}, \eta^{g}}^{C_{1}^{\sigma}, C_{2}, \ldots, C_{g}} & \sum_{c_{1} \in T_{s_{1}}^{C_{1}}} \prod_{\left(c_{2}, \ldots, c_{g}\right) \in T_{\eta^{g-1}}^{C_{2}, \ldots, C_{g}}} \prod_{i=0}^{|R|-1} s_{1 i} ! \\
& \#\left\{c_{1}^{\prime} \in R^{n} \mid \operatorname{comp}\left(c_{1}^{\prime}\right)=s_{1}, \eta^{g}\left(c_{1}^{\prime}, c_{2}, \ldots, c_{g}\right)=\eta^{g}\right\} \\
= & A_{s_{1}}^{C_{1}} A_{\eta^{g-1}}^{C_{2}, \ldots, C_{g}} \prod_{i=0}^{|R|-1} s_{1 i} ! \prod_{b \in R^{g-1}} \frac{\left(\eta_{b}^{g-1}\right) !}{\left(\eta_{\left(\omega_{0} ; b\right)}^{g}\right) ! \ldots\left(\eta_{\left(\omega_{|R|-1} ; b\right)}^{g}\right) !} .
\end{aligned}
$$

Now it is easy to complete the proof by following similar arguments stated in the proof of Theorem 3.1.

\section{The Average of Intersection Numbers}

The notion of the average intersection number was introduced in [12] for binary linear codes. We take the same notion for $R$-linear codes $C$ and $D$ of length $n$ and define the average intersection number as follows:

$$
\Delta(C, D):=\frac{1}{n !} \sum_{\sigma \in \mathcal{S}_{n}}\left|C \cap D^{\sigma}\right|
$$

Now we have the following result. 
Proposition 5.1. Let $C, D$ be two R-linear code of length $n$, and $r$ be the composition of $n$. Then we have

$$
\Delta(C, D)=\sum_{r} \frac{A_{r}^{C} A_{r}^{D}}{\left(\begin{array}{c}
n \\
r_{0}, \ldots, r_{|R|-1}
\end{array}\right)} .
$$

Proof. Let $T_{r}^{C}$ and $T_{r}^{D}$ be the set of all elements of $C$ and $D$, respectively, with the composition $r=\left(r_{0}, \ldots, r_{|R|-1}\right)$ of $n$. Then we can write

$$
\begin{aligned}
n ! \Delta(C, D) & =\sum_{\sigma \in \mathcal{S}_{n}}\left|C \cap D^{\sigma}\right| \\
& =\#\left\{(u, v, \sigma) \in C \times D \times \mathcal{S}_{n} \mid u=v^{\sigma}\right\} \\
& =\sum_{r} \sum_{u \in T_{r}^{C}} \sum_{v \in T_{r}^{D}} \#\left\{\sigma \in \mathcal{S}_{n} \mid u=v^{\sigma}\right\} \\
& =\sum_{r} A_{r}^{C} A_{r}^{D} \prod_{i=0}^{|R|-1} r_{i} ! .
\end{aligned}
$$

Hence, this completes the proof.

Let $C \subseteq \mathbb{F}_{q}^{n}$ for $q=2,3,4$ be a code. Now for $m=1,2$ we define

$$
\Delta_{J}^{m}(C):=\frac{1}{\left|J_{n}\right|} \sum_{D \in J_{n}}|C \cap D|^{m},
$$

where $J_{n}$ denotes the set of self-dual codes of Type $J$, where $J$ stands for I, II, III or IV. The following results for $J=$ I and II are presented in [13].

Theorem 5.1 ([13]). Let $C$ be a binary self-dual code of length $n$. Then

(i) $\Delta_{\mathrm{I}}(C) \approx 4$ if $C$ is of Type $\mathrm{I}$,

(ii) $\Delta_{\text {II }}(C) \approx 6$ if $C$ is of Type II.

Theorem 5.2 ([13]). Let $C$ be a binary self-dual code of length $n$. Then

(i) $\Delta_{\mathrm{I}}^{2}(C) \approx 24$ if $C$ is of Type $\mathrm{I}$,

(ii) $\Delta_{\text {II }}^{2}(C) \approx 60$ if $C$ is of Type II.

In this section, we give the analogous results of the above theorems for Type III and Type IV codes over $\mathbb{F}_{3}$ and $\mathbb{F}_{4}$ respectively. Before presenting our findings, we adopt the following mass formulas which give the numbers of Type III and Type IV codes over $\mathbb{F}_{3}$ and $\mathbb{F}_{4}$ respectively.

Theorem 5.3 ([7, 9]). The following hold: 
(i) The number of Type III codes over $\mathbb{F}_{3}$ of length $n \equiv 0(\bmod 4)$ is

$$
2 \prod_{i=1}^{n / 2-1}\left(3^{i}+1\right) .
$$

(ii) The number of Type IV codes over $\mathbb{F}_{4}$ of length $n \equiv 0(\bmod 2)$ is

$$
\prod_{i=0}^{n / 2-1}\left(2^{2 i+1}+1\right)
$$

Let $C^{\prime} \subseteq \mathbb{F}_{3}^{n}$ be a self-orthogonal code of dimension $k$. We denote by $N_{n, k}^{\mathrm{III}}$ the number of Type III codes over $\mathbb{F}_{3}$ of length $n$ containing $C^{\prime}$. Then from [1] we have

$$
N_{n, k}^{\mathrm{III}}=2 \prod_{i=1}^{n / 2-k-1}\left(3^{i}+1\right) .
$$

For $k=1$, we get from [10] the number of Type III codes over $\mathbb{F}_{3}$ of length $n$ containing a self-orthogonal vector of $\mathbb{F}_{3}^{n}$.

Now if $C^{\prime} \subseteq \mathbb{F}_{4}^{n}$ is a self-orthogonal code having dimension $k$, then the number of Type IV codes over $\mathbb{F}_{4}$ of length $n$ containing $C^{\prime}$, denoted by $N_{n, k}^{\mathrm{IV}}$, is given in [4] as follows:

$$
N_{n, k}^{\mathrm{IV}}=\prod_{i=0}^{n / 2-k-1}\left(2^{2 i+1}+1\right)
$$

In particular, for $k=1$ we get the number from [7].

The following theorem is a Type III analogue of Theorem 5.1 and Theorem 5.2,

Theorem 5.4. Let $C$ be a Type III code over $\mathbb{F}_{3}$ of length $n \equiv 0$ $(\bmod 4)$. Then we have
(i) $\Delta_{\text {III }}(C)=4-\frac{4}{3^{n / 2-1}+1} \approx 4$,
(ii) $\Delta_{\text {III }}^{2}(C)=\frac{40\left(3^{n / 2}\right)^{2}}{\left(3^{n / 2}+3\right)\left(3^{n / 2}+9\right)} \approx 40$. 
Proof. (i) Let $C \in \mathrm{III}_{n}$. Then

$$
\begin{aligned}
\sum_{D \in \mathrm{III}_{n}}|C \cap D| & =\#\left\{(u, D) \in C \times \mathrm{III}_{n} \mid u \in D\right\} \\
& =\sum_{u \in C} \#\left\{D \in \mathrm{III}_{n} \mid u \in D\right\} \\
& =\left(\sum_{u=0}+\sum_{u \in C \backslash\{0\}}\right) \#\left\{D \in \mathrm{III}_{n} \mid u \in D\right\} \\
& =\left|\mathrm{III}_{n}\right|+(|C|-1) N_{n, 1}^{\mathrm{III}} .
\end{aligned}
$$

Since $\left|\operatorname{III}_{n}\right|=2 \prod_{i=1}^{n / 2-1}\left(3^{i}+1\right)$, therefore we can write

$$
\begin{aligned}
\Delta_{\mathrm{III}}(C) & =1+(|C|-1) \frac{N_{n, 1}^{\mathrm{III}}}{\left|\mathrm{III}_{n}\right|} \\
& =1+\frac{3^{n / 2}-1}{3^{n / 2-1}+1} \\
& =\frac{3^{n / 2-1}+3^{n / 2}}{3^{n / 2-1}+1} \\
& =\frac{3^{n / 2-1}+3.3^{n / 2-1}}{3^{n / 2-1}+1} \\
& =\frac{4.3^{n / 2-1}}{3^{n / 2-1}+1} \\
& =4-\frac{4}{3^{n / 2-1}+1} .
\end{aligned}
$$

This completes the proof of (i).

(ii) Similarly as (i) we can write

$$
\begin{aligned}
\sum_{D \in \mathrm{III}_{n}}|C \cap D|^{2} & =\#\left\{(u, v, D) \in C \times C \times \mathrm{III}_{n} \mid u, v \in D\right\} \\
& =\sum_{u, v \in C} \#\left\{D \in \mathrm{III}_{n} \mid\langle u, v\rangle \subseteq D\right\} \\
& =\left(\sum_{u, v=0}+\sum_{\operatorname{dim}\langle u, v\rangle=1}+\sum_{\operatorname{dim}\langle u, v\rangle=2}\right) \#\left\{D \in \mathrm{III}_{n} \mid\langle u, v\rangle \subseteq D\right\} \\
& =\left|\operatorname{III}_{n}\right|+4(|C|-1) N_{n, 1}^{\mathrm{III}}+(|C|-1)(|C|-3) N_{n, 2}^{\mathrm{III}} .
\end{aligned}
$$


Since $\left|\operatorname{III}_{n}\right|=2 \prod_{i=1}^{n / 2-1}\left(3^{i}+1\right)$, therefore we can write

$$
\begin{aligned}
\Delta_{\mathrm{III}}^{2}(C) & =1+4(|C|-1) \frac{N_{n, 1}^{\mathrm{III}}}{\left|\mathrm{III}_{n}\right|}+(|C|-1)(|C|-3) \frac{N_{n, 2}^{\mathrm{III}}}{\left|\mathrm{III}_{n}\right|} \\
& =1+\frac{4\left(3^{n / 2}-1\right)}{3^{n / 2-1}+1}+\frac{\left(3^{n / 2}-1\right)\left(3^{n / 2}-3\right)}{\left(3^{n / 2-2}+1\right)\left(3^{n / 2-1}+1\right)} \\
& =1+\frac{12\left(3^{n / 2}-1\right)}{3^{n / 2}+3}+\frac{27\left(3^{n / 2}-1\right)\left(3^{n / 2}-3\right)}{\left(3^{n / 2}+9\right)\left(3^{n / 2}+3\right)} \\
& =\frac{40\left(3^{n / 2}\right)^{2}}{\left(3^{n / 2}+3\right)\left(3^{n / 2}+9\right)} .
\end{aligned}
$$

This completes the proof of (ii).

We close this paper with the following Type IV analogue of Theorem 5.1 and Theorem 5.2 .

Theorem 5.5. Let $C$ be a Type IV code over $\mathbb{F}_{4}$ of length $n \equiv 0$ $(\bmod 2)$. Then we have

(i) $\Delta_{\mathrm{IV}}(C)=3-\frac{3}{2^{2(n / 2)-1}+1} \approx 3$,

(ii) $\Delta_{\mathrm{IV}}^{2}(C)=\frac{27\left(2^{2(n / 2)}\right)^{2}}{\left(2^{2(n / 2)}+2\right)\left(2^{2(n / 2)}+8\right)} \approx 27$.

Proof. (i) Let $C \in \mathrm{IV}_{n}$. Then

$$
\begin{aligned}
\sum_{D \in \mathrm{IV}_{n}}|C \cap D| & =\#\left\{(u, D) \in C \times \mathrm{IV}_{n} \mid u \in D\right\} \\
& =\sum_{u \in C} \#\left\{D \in \mathrm{IV}_{n} \mid u \in D\right\} \\
& =\left(\sum_{u=0}+\sum_{u \in C \backslash\{0\}}\right) \#\left\{D \in \mathrm{IV}_{n} \mid u \in D\right\} \\
& =\left|\mathrm{IV}_{n}\right|+(|C|-1) N_{n, 1}^{\mathrm{IV}} .
\end{aligned}
$$


Since $\left|\mathrm{IV}_{n}\right|=\prod_{i=0}^{n / 2-1}\left(2^{2 i+1}+1\right)$, therefore,

$$
\begin{aligned}
\Delta_{\mathrm{IV}}(C) & =1+(|C|-1) \frac{N_{n, 1}^{\mathrm{IV}}}{\left|\mathrm{IV}_{n}\right|} \\
& =1+\frac{2^{2(n / 2)}-1}{2^{2(n / 2)-1}+1} \\
& =\frac{2^{2(n / 2)-1}+2^{2(n / 2)}}{2^{2(n / 2)-1}+1} \\
& =\frac{3.2^{2(n / 2)-1}}{2^{2(n / 2)-1}+1} \\
& =3-\frac{3}{2^{2(n / 2)-1}+1}
\end{aligned}
$$

This completes the proof of (i).

(ii) Similarly as (i) we can write

$$
\begin{aligned}
\sum_{D \in \mathrm{IV}_{n}}|C \cap D|^{2} & =\#\left\{(u, v, D) \in C \times C \times \mathrm{IV}_{n} \mid u, v \in D\right\} \\
& =\sum_{u, v \in C} \#\left\{D \in \mathrm{IV}_{n} \mid\langle u, v\rangle \subseteq D\right\} \\
& =\left(\sum_{u, v=0}+\sum_{\operatorname{dim}\langle u, v\rangle=1}+\sum_{\operatorname{dim}\langle u, v\rangle=2}\right) \#\left\{D \in \mathrm{IV}_{n} \mid\langle u, v\rangle \subseteq D\right\} \\
& =\left|\mathrm{IV}_{n}\right|+5(|C|-1) N_{n, 1}^{\mathrm{IV}}+(|C|-1)(|C|-4) N_{n, 2}^{\mathrm{IV}} .
\end{aligned}
$$

Since $\left|\mathrm{IV}_{n}\right|=\prod_{i=0}^{n / 2-1}\left(2^{2 i+1}+1\right)$, therefore,

$$
\begin{aligned}
\Delta_{\mathrm{IV}}^{2}(C) & =1+5(|C|-1) \frac{N_{n, 1}^{\mathrm{IV}}}{\left|\mathrm{IV}_{n}\right|}+(|C|-1)(|C|-4) \frac{N_{n, 2}^{\mathrm{IV}}}{\left|\mathrm{IV}_{n}\right|} \\
& =1+5 \frac{2^{2(n / 2)}-1}{2^{2(n / 2)-1}+1}+\frac{\left(2^{2(n / 2)}-1\right)\left(2^{2(n / 2)}-4\right)}{\left(2^{2(n / 2)-3}+1\right)\left(2^{2(n / 2)-1}+1\right)} \\
& =1+10 \frac{2^{2(n / 2)}-1}{2^{2(n / 2)}+2}+16 \frac{\left(2^{2(n / 2)}-1\right)\left(2^{2(n / 2)}-4\right)}{\left(2^{2(n / 2)}+8\right)\left(2^{2(n / 2)}+2\right)} \\
& =\frac{27\left(2^{2(n / 2)}\right)^{2}}{\left(2^{2(n / 2)}+8\right)\left(2^{2(n / 2)}+2\right)} .
\end{aligned}
$$

This completes the proof of (ii). 


\section{ACKNOWLEDGEMENTS}

The authors thank Manabu Oura for helpful discussions. The authors would also like to thank the anonymous reviewers for their beneficial comments on an earlier version of the manuscript. The second named author is supported by JSPS KAKENHI (18K03217).

\section{REFERENCES}

[1] A. Bassa, and N. Tutas, Extending self-orthogonal codes, Turk. J. Math. 43 (2019), 2177-2182.

[2] H. S. Chakraborty, and T. Miezaki, Variants of Jacobi polynomials in coding theory, in preparation.

[3] H. S. Chakraborty, T. Miezaki, and M. Oura, On the cycle index and the weight enumerator II, submitted.

[4] J. H. Conway, and V. Pless, Self-dual codes over $G F(3)$ and $G F(4)$ of length not exceeding 16, IEEE Transactions and Information Theory IT-25(3) (1979), 312-322.

[5] S. T. Dougherty, M. Harada, and M. Oura, Note on the $g$-fold joint weight enumerators of self-dual codes over $\mathbb{Z}_{k}$, Applicable Algebra in Engineering, Communication and Computing 11 (2001), 437-445.

[6] F. J. MacWilliams, C. L. Mallows, and J. H. Conway, Generalizations of Gleason's theorem on weight enumerators of self-dual codes, IEEE Trans. Information Theory IT-38 (1972), 794-805.

[7] F. J. MacWilliams, A. M. Odlyzko, N. J. A. Sloane, and H. N. Ward, Self-dual codes over GF(4), J. Comb. Theory 25A (1978), 288-318.

[8] T. Miezaki, and M. Oura, On the cycle index and the weight enumerator, Des. Codes Cryptogr. 87 (2019), no. 6, 1237-1242.

[9] V. Pless, On the uniqueness of the Golay codes, J. Comb. Theory 5 (1968), 215-228.

[10] V. Pless, and J. N. Pierce, Self-dual codes over $G F(q)$ satisfy a modified Varshamov-Gilbert bound., Information and Control 23 (1973), 35-40.

[11] I. Siap, and D. K. Ray-Chaudhuri, On $r$-fold complete weight enumerator of $r$ linear codes, in: Contemp. Math. American Math. Society, 259, (2000), 501-513.

[12] T. Yoshida, The average of joint weight enumerators, Hokkaido Mathematical Journal 18 (1989), 217-222.

[13] T. Yoshida, The average intersection number of a pair of self-dual codes, Hokkaido Mathematical Journal 20 (1991), 539-548.

(1) Graduate School of Natural Science and Technology, Kanazawa University, Ishikawa 920-1192, Japan, (2) Department of Mathematics, Shahjalal University of Science and Technology, Sylhet-3114, BANGLADESH,

Email address: himadri-mat@sust.edu

Faculty of Education, University of the Ryukyus, Okinawa 903-0213, JAPAN,

Email address: miezaki@edu.u-ryukyu.ac.jp 